\title{
Transvenous ablation of atrioventricular conduction with a low energy power source
}

\author{
EDWARD ROWLAND, DAVID CUNNINGHAM, ARIF AHSAN, \\ ANTHONY RICKARDS \\ From the National Heart and Lung Institute and National Heart and Chest Hospitals, London
}

SUMMARY A power source modified to increase voltage delivery and minimise arcing (for a given energy) was used for transvenous ablation of atrioventricular conduction to control refractory supraventricular arrhythmias in 14 patients. Twelve had atrial fibrillation or flutter, one had atrioventricular nodal reentry tachycardia, and the other had permanent junctional reentry tachycardia. Despite treatment with 5-7 (median 6) antiarrhythmic drugs symptoms had persisted in all the patients. Cathodal discharges of 0.5-39.5 J were delivered to the distal electrode (in one case in parallel with the middle electrode). In all patients shocks produced complete atrioventricular block; this was permanent in eleven (79\%). Four patients required a second procedure. In one patient, only a transient atrioventricular block could be produced and catheter ablation with a conventional power source also failed. In the other two atrioventricular conduction was modified such that previously ineffective treatment produced satisfactory control of heart rate. The cumulative energy delivered to those in whom permanent complete heart block resulted ranged from 3.6 to 97.8 (mean $38 \cdot 3$ ) J with a mean of three shocks (range 1-7) delivered per patient. During follow up of 1-28 (mean 14) months 11 patients remained in complete heart block and free of arrhythmia.

Transvenous ablation of atrioventricular conduction conventionally uses high energy discharges generated by a standard defibrillator and has become an accepted and effective method of creating complete atrioventricular block in patients with refractory supraventricular arrhythmias. ${ }^{1-4}$ The precise mechanism by which these high energies destroy the atrioventricular conduction system is unknown but high energy discharges produce an explosive flash (arcing) that is associated with a raised local temperature and a high intensity pressure wave. ${ }^{56}$ Examination of the Ablation Registry showed that this technique achieved complete heart block in $63 \%$ of patients and that the mean cumulative stored energy required was $611 \mathrm{~J} .{ }^{4}$ The recommended conventional technique uses one or more shocks of 150 $300 \mathrm{~J}$ delivered between one electrode of a standard $6 \mathrm{~F}$ or $7 \mathrm{~F}$ pacing catheter and a back plate. ${ }^{7}$ Several adverse effects have been reported including myocar-

Requests for reprints to Dr Edward Rowland, National Heart Hospital, Westmoreland Street, London W1M 8BA.

Accepted for publication 2 May 1989 dial rupture, ${ }^{8}$ and there is concern about intravascular haemolysis ${ }^{9}$ and right ventricular dysfunction, ${ }^{10}$ and that gas bubbles may be produced in both ventricles immediately after the shock. ${ }^{11}$ Although it is not clear which properties of the high energy cause the desired clinical effect it seems likely that many of these adverse effects result from the pressure wave.

We have designed a power source that shortens the energy delivery tume in order to produce shocks that increase peak voltage and current for a given energy. ${ }^{12}$ Our previous work has shown that this energy source has a higher arcing threshold than a standard defibrillator, with the result that local electrical effects predominate over those resulting from arcing (barotrauma). ${ }^{13}$

\section{Patients and methods}

Fourteen patients with drug refractory supraventricular arrhythmias underwent attempted transvenous ablation of atrioventricular conduction. Twelve had paroxysmal or established atrial fibrillation or flutter, one had paroxysmal atrioventricular nodal 
Table Data on patients who had ablation of the atrioventricular pathway by the new ablater

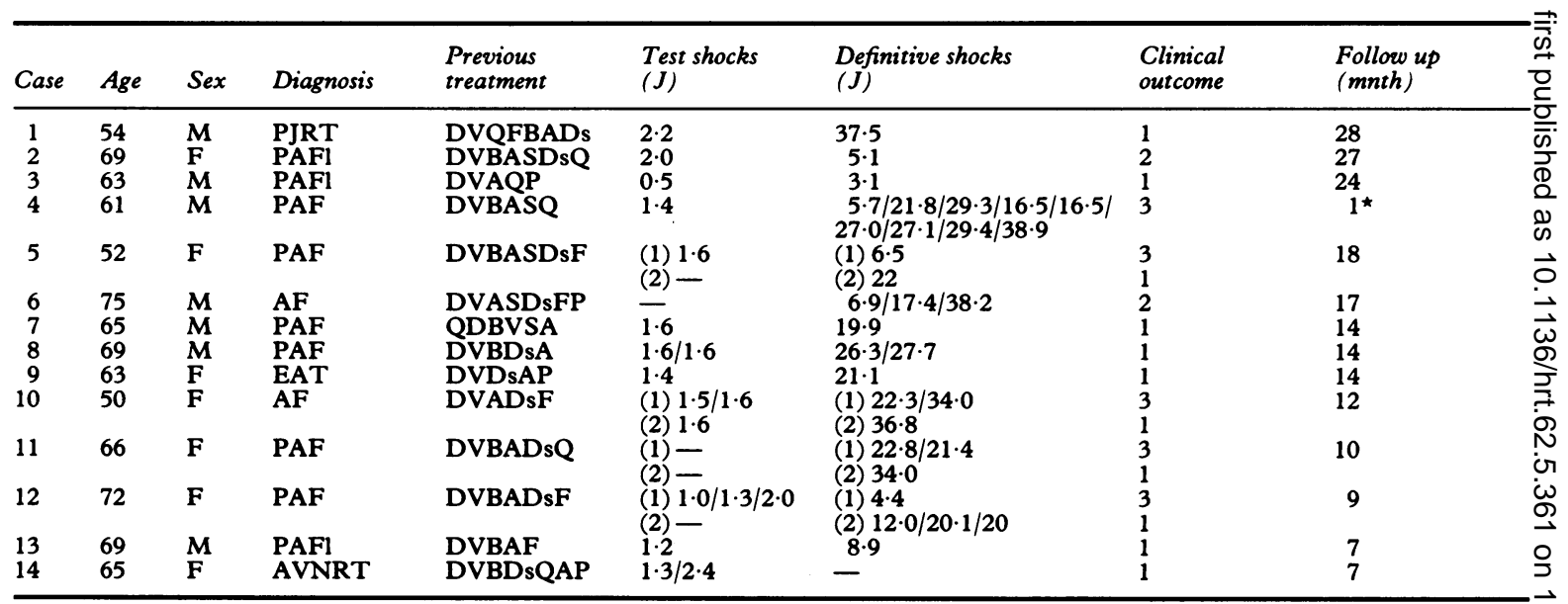

Diagnosis: PJRT, permanent junctional reentry tachycardia; PAFl, paroxysmal atrial flutter; PAF, paroxysmal atrial fibrillation; EAT, Z ectopic atrial tachycardia; AF, established atrial fibrillation; AVNRT, atrioventricular nodal reentry tachycardia.

Drugs: D, digoxin; V, verapamil; Q, quinidine; B, $\beta$ blockers; Ds, disopyramide; F, flecainide; S, sotalol; P, propafenone; $A$, amiodarone.

All energies are the delivered values. Where second procedures were performed the values are indicated for the first (1) and second (2) procedures.

Clinical outcome: 1 , complete heart block; 2 , impaired atrioventricular conduction; 3 , no effect.

* Surgical ablation of atrioventricular conduction.

reentry tachycardia, and one had the permanent form of junctional reentry tachycardia. All patients had received at least five antiarrhythmic drugs, including amiodarone, which had either been ineffective or had been stopped because of side effects (table). None had undergone previous transvenous ablation. Antiarrhythmic drugs were stopped before the procedure in 11 patients-the other three were continued on atrial stabilising drugs to prevent atrial arrhythmias during ablation.

\section{PROCEDURE}

After informed consent had been obtained and premedication given a small (4F or $5 \mathrm{~F}$ ) pacing electrode was introduced percutaneously via the right subclavian vein, positioned at the apex of the right ventricle, and used for temporary ventricular pacing. The His bundle electrogram was recorded with a 7F USCI tripolar electrode in 13 cases (a 6F tripolar electrode was used in the other) and positioned adjacent to the tricuspid valve. Three patients were in atrial fibrillation at the time of the procedure and an adequate $(\geqslant 200 \mu \mathrm{V})$. His bundle could not be identified; external DC cardioversion was performed in order to restore sinus rhythm. In these a third electrode was used for temporary atrial pacing to maintain atrial stability after DC cardioversion.

Bipolar and unipolar electrograms from the tripolar electrode catheter were recorded and the position of the electrode catheter adjusted until the largest His bundle electrogram was recorded on the distal pair. Unipolar electrograms were also recorded to ensure that the distal electrode had the largest His bundle component. If the atrial electrogram on the bipolar signal was lower in amplitude than the Hisi bundle potential the catheter was withdrawn towards the atrium.

When the optimal $\mathrm{His}$ bundle electrode catheter position had been identified the patient was anaes thetised, a defibrillator plate placed under the left scapula, and the His bundle electrogram re-checked $\Phi$ A blood sample was then taken for estimation of the serum concentration of creatine kinase (total and $\mathrm{MB}$ ? fraction).

The power source used in this study was a specially? designed ablater (Cardiac Recorders CR60), the characteristics of which have been described. ${ }^{12}$ This device produces a capacitive discharge without inductance modification. We used resistive atten uators and precision differential amplifiers too measure the delivered voltage and current: then

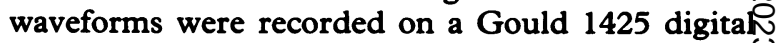
storage oscilloscope and data ( $8 \mathrm{bit}$ ) transferred on $a^{\omega}$ serial link to a microcomputer (Hewlett-Packard 150) for computation of energy, power, and impedance. All shocks were delivered synchronously? with the $\mathbf{R}$ wave of a surface electrocardiogram lead.

The cathodal output of the ablater was connectedo to the distal electrode of the His bundle electroded catheter and "test" shocks of $0.5,1$, or $2 \mathrm{~J}$ wereके selected and delivered to test the responsiveness of 
atrioventricular conduction. If right bundle branch block was produced the His bundle electrode catheter was withdrawn towards the atrium. In one case the electrode catheter prolapsed into the atrium when it was withdrawn; the electrode catheter was therefore positioned optimally and further shocks were delivered through the distal and middle electrodes which were connected in common. The response of the atrioventricular conduction system was observed for up to 25 minutes.

When complete heart block did not persist "definitive" shocks of $5,10,20,30$, or $40 \mathrm{~J}$ were then selected and delivered, the energy level chosen depending on the degree and duration of heart block produced by the test shock. Shocks were delivered in the same configuration as that used for the test shocks. The effect of the definitive shock was monitored for 25 minutes and if complete heart block persisted no further shocks were delivered. If atrioventricular conduction returned further shocks of 20,30 , or $40 \mathrm{~J}$ were administered.

The patients were observed for 24 hours and a permanent rate responsive ventricular demand pacemaker (VVI) or atrioventricular sequential pacemaker was implanted when third degree atrioventricular block persisted or when evidence of considerable impairment of atrioventricular conduction remained. In those in whom atrioventricular conduction returned to normal the ablation procedure was repeated. On this occasion test shocks were not used; an energy level above that used at the first ablation attempt was selected (that is, 20,30 , or $40 \mathrm{~J})$.

After each ablation procedure blood was collected for estimation of creatine kinase at eight and 24 hours.

\section{Results}

Permanent complete heart block was achieved in 11 patients, there was no change in atrioventricular conduction in one patient, and in the other two patients atrioventricular conduction was modified. Fifty shocks were delivered in the 14 patients and the mean cumulative energy delivered was 50.4 (range $3 \cdot 6-212 \cdot 2) \mathrm{J}$. Figure 1 shows the overall persistence of atrioventricular conduction in response to the shocks. In those in whom permanent complete heart block was achieved the mean cumulative energy delivered was 38.3 (range 3.6-97.7) J and the mean number of shocks required was three (range 1-7).

\section{TEST SHOCKS}

Test shocks were not used in two patients because the electrode catheter was believed to be in an unstable position and creation of a heart block was thought to

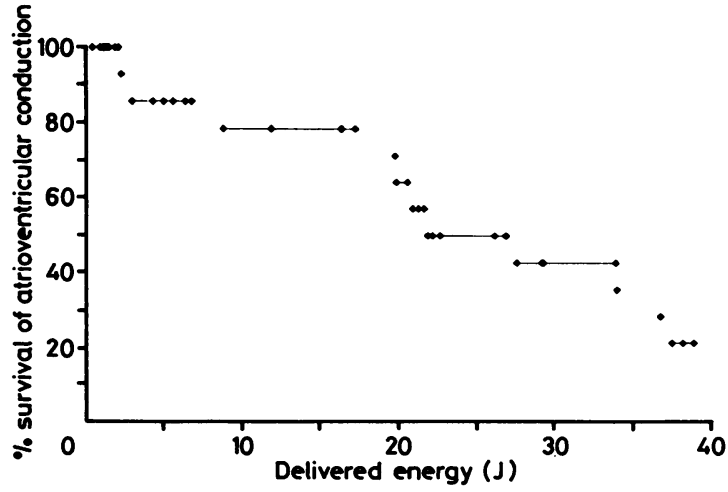

Fig 1 Survival of atrioventricular conduction in response to increasing energy. Each of the fifty shocks used is plotted. In three patients $(21 \%)$ atrioventricular conduction survived intact. For the purposes of the survival curve the highest energy shock delivered to a patient who attained permanent heart block was assumed to have been successful.

be more pressing. In one patient the test shock (delivered energy $2.4 \mathrm{~J}$ ) produced permanent complete heart block, associated after 18 hours with a narrow QRS escape rhythm of 55 beats $/ \mathrm{min}$. In two patients the test shock resulted in transient right bundle branch block and withdrawal of the His bundle electrode toward the atrium was associated with transient complete heart block when a subsequent test shock was used. Therefore in 11 patients delivered energies of $0 \cdot 5-2 \cdot 2 \mathrm{~J}$ produced transient interruption of atrioventricular conduction which lasted for 30 seconds to 23 minutes.

\section{DEFINITIVE SHOCKS}

Between one and nine shocks ranging from 3.1 to $38.9 \mathrm{~J}$ were delivered in 13 patients. In six permanent complete heart block was achieved with a single definitive shock (delivered energy 3.1-37.5 J) (fig 2). In one patient only transient interruption of atrioventricular conduction could be achieved. In this patient the His bundle electrogram remained small $(100 \mu \mathrm{V})$, despite repositioning of the electrode catheter, and repeated shocks of increasing intensity in various positions did not interrupt atrioventricular conduction. Electrode catheter ablation performed subsequently with a conventional power source (300 J shocks) was equally ineffective. In the remaining six patients transient atrioventricular block resulted from the first definitive shock and one or two further shocks were required to produce complete heart block for the duration of the observation period.

Four patients had a second ablation procedure performed because complete heart block did not 


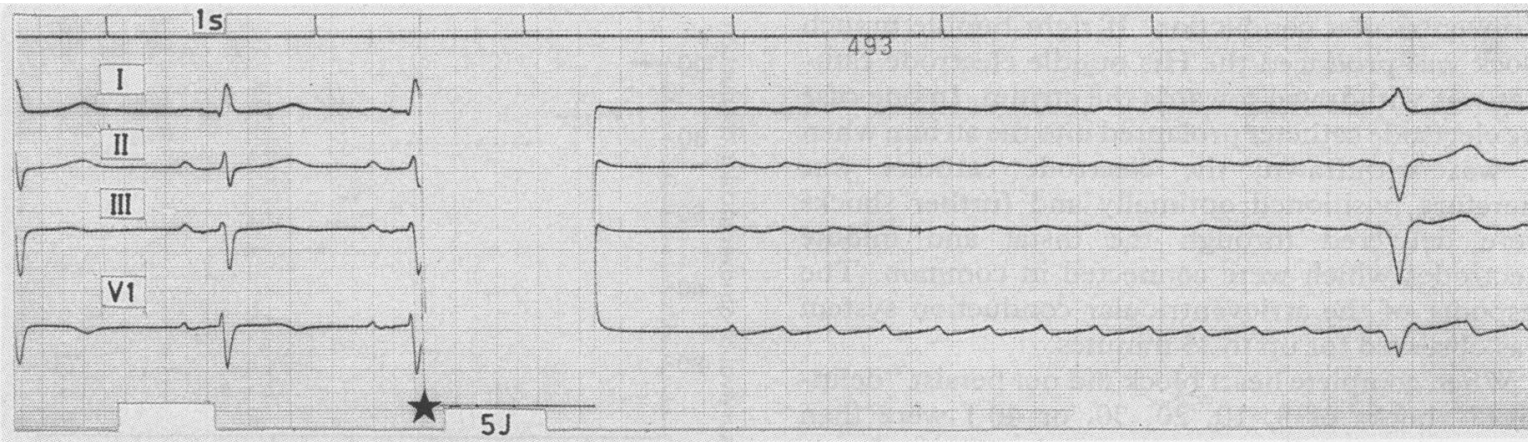

Fig 2 In patient 2 (a woman of 69) a synchronised shock of $5 \mathrm{~J}\left({ }^{\star}\right.$ ) was delivered during sinus rhythm and resulted in complete heart block, with simultaneous induction of atrial flutter. Electrocardiographic leads I, II, III, and VI are shown.

persist after the first attempt and atrioventricular conduction returning within 2-6 hours. Complete heart block was achieved in all of them at the second procedure.

In three patients atrial fibrillation was induced by one of the ablation shocks but non-sustained or sustained ventricular tachycardia or ventricular fibrillation was not induced in any of them.

In 11 patients serum concentrations of creatine kinase were measured before and after the procedure and in two there was a significant rise, but both patients had had multiple transthoracic cardioversions to establish sinus rhythm during the ablation procedure. In none of the patients was there a significant increase in the creatine kinase MB fraction.

\section{FOLLOW UP}

Complete heart block was maintained in 11 patients during follow up of 7-28 (mean 14) months. In each patient either a rate responsive ventricular demand (10 patients) or atrioventricular sequential pacemaker was implanted. None of these patients is being treated with antiarrhythmic agents and none has symptomatic palpitation. One patient had an uncomplicated anterior myocardial infarction 11 months after the ablation and another experienced an episode of persistent chest pain five months later but had no enzyme evidence of infarction. Surgical ablation of the His bundle was successful in the patient in whom both modified and conventional ablation failed.

In two patients atrioventricular conduction seemed to return late after the ablation but when it did there was evidence of impaired conduction. In patient 6 atrial fibrillation recurred 24 hours after the procedure and was conducted at a mean ventricular rate of 68 beats/minute compared with 122 beats/ minute before ablation. Although the resting and exercise heart rates during atrial fibrillation subsequently increased they remained well controlled on digoxin. Before ablation they had $\stackrel{\oplus}{\oplus}$ remained uncontrolled despite combined treatments with digoxin and verapamil. This patient was notpaced and repeated ambulatory electrocardiographicz recordings did not show prolonged pauses. The other patient who presented with paroxysmal atrial flutter, 3 was in complete heart block after a $5.1 \mathrm{~J}$ dischargeo and this was thought to be present at the end of the observation period. Closer analysis showed that thereo was high degree (6:1) atrioventricular block at the end of the procedure; subsequently paroxysms of atrial flutter were conducted slowly. A symptomatic s episode of palpitation recurred, however, six monthso after the ablation and atrial flutter with $2: 1$ atrio-o ventricular block was documented. Treatment with digoxin was started and further attacks of atria $\overrightarrow{5}$ flutter were associated with high degree atrio- $\exists$ ventricular block.

\section{Discussion}

Electrode catheter ablation of atrioventricular conduction is used to treat patients with supra-i ventricular arrhythmias who continue to have symptoms while taking medical treatment or who are intolerant of medical treatment. Data collected by the Ablation Registry showed that permanent completeo heart block was produced in $63 \%$ of 360 patients who had ablation of the atrioventricular tract (mean $611 \mathrm{~J} / \mathrm{s}$ patient). ${ }^{4}$ Similar results were reported from tho United Kingdom and France. ${ }^{314}$ Complications may however, follow the use of these high energy shocks Ventricular arrhythmias may occur in the short term as may hypotension and cardiac perforation. ${ }^{4-8}$ Thereis a short lived rise in cardiac enzymes ${ }^{1-4}$ and evidences? that right ventricular function may be impaired in the long term. ${ }^{10}$ Follow up of the registry patients also showed that $2 \%$ died suddenly in the mean follow up period of nine months."

A defibrillator is conventionally used as the power 
source for catheter ablation, despite the fact that to minimise injury to the chest wall it has a waveform that is designed to limit the peak current for a given energy. Because the intention of catheter ablation is to cause localised damage this waveform may not be appropriate.

Several physical events result from the discharge of a high voltage through a small intracardiac electrode. ${ }^{56}$ The precise physical features of the energy that cause damage are unknown. After discharge of the defibrillator an intense electric field surrounds the intracardiac electrode and current flow results in resistive heating. With all but the lowest defibrillator energies arcing will occur across the gas that builds up around the electrode as a consequence of this local heating. Arcing will result in a high pressure shock wave that will propagate without local attenuation. ${ }^{15}$ The series inductance in the output circuit of a standard defibrillator retards the rate of discharge of the capacitor and increases the time to peak voltage. This allows a high voltage to be present when insulating gas has formed around the electrode and therefore arc formation is encouraged. The modified ablater was designed to deliver similar voltage but over a shorter interval, in part to allow delivery of the maximum amount of energy before the build up of gas. This design permits higher voltage and current to be delivered for a given energy and allows the local effects of cellular electric field dielectric breakdown, ${ }^{16}$ ionisation, and heating to predominate over barotrauma. ${ }^{12}$ It is likely that the rise in temperature is limited to such an extent that it does not produce any important tissue damage.

Our results indicate that electrode catheter ablation of atrioventricular conduction is successful in a high percentage of patients at considerably lower energies than previously reported. We measured the delivered voltage and current and were therefore able to compute delivered energy. Such measurements have not been systematically reported before and therefore comparisons with other series cannot easily be made. In most previous studies only the stored energies or the energy delivered to a nominal resistance were recorded. It is difficult, therefore, to establish the relation between the voltages and currents associated with the energies used in this study and those seen with conventional defibrillator shocks. Trantham et al, however, did record voltage and current in 12 patients undergoing electrode catheter ablation by conventional shocks of 200 and $300 \mathrm{~J}$-the mean peak voltages were 2.16 and $2.40 \mathrm{kV}$ and the mean peak currents were 42.2 and $58.2 \mathrm{~A}$ respectively. ${ }^{17}$ In those patients in our series in whom a single definitive shock caused permanent complete heart block, the mean energy was 16.4 (range $2.4-37.5$ ) $\mathrm{J}$, the mean peak voltage 1.85 (range
$0 \cdot 79-2 \cdot 78) \mathrm{kV}$, and the mean peak current 15.5 (range 4.6-25) A.

We used standard electrodes and techniques for His bundle recording as well as standard criteria for determining the optimal site for energy delivery. Other studies have also attempted to reduce the energy requirements for electrode catheter ablation of atrioventricular conduction. Holt et al used the specially shaped design of helical permanent pacing electrodes to ensure both a closer contact with the target and direct the energy. ${ }^{18}$ Four shocks of $50 \mathrm{~J}$ each in 10 patients achieved complete heart block in seven. Polgar et al used a suction electrode to obtain close contact with the His bundle and achieved complete heart block in three of five patients with single shocks of $50-150 \mathrm{~J} .{ }^{19}$ Although the serum concentration of creatine kinase was raised the concentration was lower than in a comparative group undergoing atrioventricular ablation in the conventional method. McComb et al attempted electrode catheter ablation of atrioventricular conduction in seven patients at low levels of energy $(20-50 \mathrm{~J})$ from a defibrillator in an attempt to impair but not destroy atrioventricular conduction..$^{20}$ Single initial shocks of $50 \mathrm{~J}$, however, produced permanent complete heart block in three patients.

The absence of a rise in cardiac enzymes in our patients is intriguing. The peak voltages (and associated peak currents) seen in our patients are equivalent to energies 5-10 times higher when a standard defibrillator is used. Therefore the electric field intensities are similar to those encountered when the conventional technique is used and cardiac enzymes are increased. ${ }^{17}$ If as seems possible myocardial damage is produced as a consequence of the diffuse effect of a shock wave, possibly by means of cavitation, this provides further circumstantial evidence that this modified waveform reduces the arcing tendency and results in a lower pressure shock wave for a given peak voltage.

Our protocol for determining the efficacy of individual shocks differed from reports of other methods. We used test shocks in an attempt to determine whether the catheter was positioned at the correct level of the conduction system and additionally to provide a guide to the responsiveness of atrioventricular conduction to electrical discharge. It is clear that the energy-response relation with our technique is wide and presumably influenced by the proximity of the electrode to the conducting system. Whether the amplitude of the His bundle electrogram is a useful guide to proximity remains controversial. In some studies it correlated with clinical efficacy $^{2}$ while in others no relation was found. ${ }^{3}$ Clearly no individual value for the amplitude of the His bundle potential will determine successful 
catheter ablation. We attempted on each occasion to position the electrode catheter so that it recorded an amplitude $>200 \mu \mathrm{V}$, and in two of the three patients in whom complete heart block was not achieved the His bundle potential was $<200 \mu \mathrm{V}$.

Test shocks may dislodge the electrode. If this happens the catheter will have to be repositioned while the $\mathrm{His}$ bundle is temporarily disrupted and therefore not producing as large an electrical signal. In fact movement of the electrode catheter was slight at these low energies, but during the shocks and for the observation period afterwards the operator held the catheter to maintain its position. If dislodgement had been an important problem we would have expected a higher incidence of repeat procedures. But only four (29\%) of 14 required second ablations compared with $37 \%$ and $58 \%$ in larger series. ${ }^{34}$

This study showed that permanent ablation of atrioventricular conduction can be achieved with a purpose built ablater. This power source achieved complete heart block with cumulative energies that were several times lower than those required with a conventional defibrillator. This power source, which was designed to optimise the local electrical effects and reduce the tendency to arcing, gave results that suggested that arcing is not essential for the ablation of atrioventricular conduction. We do not know whether avoidance of arcing and its attendant effects will improve the long term outcome of patients undergoing ablation.

\section{References}

1 Scheinman MM, Morady F, Hess S, Gonzalez R. Catheter-induced ablation of the atrioventricular junction to control refractory supraventricular arrhythmias. JAMA 1982;248:851-5.

2 Gallagher JJ, Svenson RH, Kasell JH, et al. Catheter technique for closed-chest ablation of the atrioventricular conduction system: a therapeutic alternative for the treatment of refractory SVT. $N$ Engl J Med 1982;306:194-200.

3 Nathan AW, Bennett DH, Ward DE, Camm AJ. Catheter ablation of atrioventricular conduction. Lancet 1984;i:1280-4.

4 Evans GT, Scheinman MM. Catheter ablation of ventricular tachycardia foci: a report of the percutaneous cardiac mapping and ablation registry [Abstract]. Circulation 1986;74(suppl II):460.

5 Boyd EGCA, Holt PM. An investigation into the electrical ablation technique and a method of electrode assessment. PACE 1985;8:815-23.

6 Bardy GH, Coltorti F, Ivey TD, et al. Some factors affecting bubble formation with catheter-mediateddefibrillator impulses. Circulation 1986;73:525-38. $\overrightarrow{\bar{F}}$

7 Scheinman MM. Catheter ablation for patients with? cardiac arrhythmias. $P A C E$ 1986;9:551-64.

8 Fisher JD, Kim SG, Matos JA, et al. Complications of catheter ablation of tachyarrhythmias: occurrence protection and prevention. Clin Prog Electrophysio Pacing 1985;3:292-8.

9 Holt PM, Boyd EGCA. Hematologic effects of the high-energy endocardial ablation technique $\overrightarrow{0}$ Circulation 1986;73:1029-36.

10 Schofield PM, Bowes RJ, Brooks N, Bennett DH $\vec{\omega}$ Exercise capacity and spontaneous heart rhythm after? transvenous fulguration of atrioventricular conduc tion. Br Heart J 1986;56:358-65.

11 Rowland E, Foale R, Nihoyannopoulos P, Perelman $M_{i r}^{N}$ Krikler DM. Intracardiac contrast echoes duringu transvenous His bundle albation. $\mathrm{Br}$ Heart $\mathcal{\rho}$ 1985;53:240-2.

12 Cunningham D, Rowland E, Rickards AF. A new low energy power source for catheter ablation. $P A C \overrightarrow{E_{Z}}$ 1986;9:1384-90.

13 Ahsan AJ, Cunningham D, Rowland E, Rickards AFळ Catheter ablation without fulguration: design and performance of a new system. PACE 1989;12(Part⿱ II):131-5.

14 Lévy S, Bru P. Interruption électrique par voi $\overrightarrow{\hat{E}_{0}}$ percutanée de la conduction auriculo-ventriculairé normale. Analyse des cas français. Arch Mal Coeuঠ̃ 1986;79:1145-50.

15 Cunningham D, Rowland E, Rickards AF. Lack of shock wave absorption after high energy cathete discharge suggests that barotrauma is a myth [Abstract]. Circulation 1987;76(suppl IV):407.

16 Jones JL, Proskauer CC, Paull WK, Lepeschkin E $\overrightarrow{0}$ Jones RE. Ultrastructural injury to chick myocardiaß cells in-vitro following “electrical countershock"
Circ Res 1980;46:387-49.

17 Trantham JL, Gallagher JJ, German LD, Broughton A, Guarneri T, Kasell J. Effects of energy delivery viad a His bundle catheter during closed chest ablation of the atrioventricular conduction system. J Clin Invesi1983;72:1563-74.

18 Holt PM, Boyd EGCA, Crick J, Sowton E. Low energies and Helifix electrodes in the successfub ablation of atrioventricular conduction. $P A C E$ 1985;8:639-45.

19 Polgar P, Worum F, Kovacs P, Lorincz I, Bekassy SzP Peterffy A. A new technique for closed-chest humant. His bundle ablation using suction electrode catheter and DC shock. In: Perez-Gomez F, ed. Cardiae pacing, electrophysiology, tachyarrhythmias. New York: Futura Media Services, 1985:1582-7.

20 McComb JM, McGovern BA, Garan H, Ruskin JN Modification of atrioventricular conduction usin low energy transcatheter shocks [Abstract]. J Am Colf Cardiol 1985;5:454. 